\title{
Unusual Cystic and Papillary Appearance of Brain Metastasis from Papillary Lung Adenocarcinoma on MRI. Radiologic-Pathologic Correlations in Two Similar Cases
}

\author{
Patrick Mailleux $^{1 *}$, Marie Mailleux ${ }^{2}$, Anne-Sophie Marchand ${ }^{3}$ \\ ${ }^{1}$ Department of Imaging, Clinique St Luc, Bouge, Belgium \\ ${ }^{2}$ Department of Oncology, Clinique St Luc, Bouge, Belgium \\ ${ }^{3}$ Department of Imaging, CHU UCL Namur, Namur, Belgium \\ Email: *p.mailleux@gmail.com
}

How to cite this paper: Mailleux, P., Mailleux, M. and Marchand, A.-S. (2020) Unusual Cystic and Papillary Appearance of Brain Metastasis from Papillary Lung Adenocarcinoma on MRI. Radiologic-Pathologic Correlations in Two Similar Cases. Advances in Lung Cancer, 9, 17-23.

https://doi.org/10.4236/alc.2020.92002

Received: March 23, 2020

Accepted: April 12, 2020

Published: April 15, 2020

Copyright $\odot 2020$ by author(s) and Scientific Research Publishing Inc. This work is licensed under the Creative Commons Attribution International License (CC BY 4.0).

http://creativecommons.org/licenses/by/4.0/

\begin{abstract}
We report two cases of brain metastasis from a lung adenocarcinoma. Magnetic Resonance Imaging (MRI) appearance was closely correlated with the macroscopic and microscopic findings that showed multiples nodules of a well-differentiated papillary adenocarcinoma with secreting tubules, surrounded by mucoid fluid.
\end{abstract}

\section{Keywords}

Brain MRI, Metastasis, Lung Adenocarcinoma, Papillary

\section{Introduction}

Brain metastases are more frequent than primary brain tumors. In many cases Magnetic Resonance Imaging (MRI) is helpful to distinguish the primary tumors and abscesses from metastases. However, it usually gives no hint about the origin of the primary tumor. In the cases herein reported, the close correlation between the MRI images and the histologic appearance lets us suggest that the MRI pattern could help the diagnosis and narrow the differential diagnosis to papillary secreting adenocarcinoma.

\section{Case Report}

\subsection{Case 1}

A 57 year-old non smoking female presented impaired speech fluency and diffi- 
culty to find words for 2 weeks, without any problem of understanding. The neurological examination was otherwise normal.

Unenhanced Computed Tomography (CT) showed a large left frontal mass with a cystic portion and surrounding edema. There was neither calcification nor hemorrhage (Figure 1). On MRI, the mass was $39 \mathrm{~mm}$-large, surrounded by edema beyond a $1.4 \mathrm{~mm}$-thick regular peripheral wall, enhancing homogeneously. Inside the mass were a large peripheral fluid area and multiple nodules. Those nodules had 5 to $7 \mathrm{~mm}$ of outer diameter and demonstrated intense peripheral contrast enhancement similar to the capsule. There were cysts, and their central part seemed sometimes to be linked one with another (Figure 2). Neither hemorrhage nor calcifications were seen on gradient echo imaging. Chest Xray performed the same day showed a large right upper lobe tumor, with mediastinal lymphadenopathies on subsequent chest CT (Figure 3).

The brain tumor was resected with large margins as the surgeon found no cleavage line with the adjacent brain. A mucoid viscous fluid flowed during its opening that revealed the multiple small nodules described on MRI.

Histology (Figure 4) revealed a metastasis of a large-cell mucosecreting adenocarcinoma of a papillary type, consisting of mucus surrounding small tumoral proliferations, consisting of peripheral papillary tumoral structures with a hierarchical branching pattern: irregular papillae branch from large to progressively smaller papillae and terminating in detached tufts of epithelial cells. Tumor cells had a low mitotic activity. EGFR testing was positive in $80 \%$ of cells with grade 3 intensity.

The patient treatment started with complete surgical resection of the left frontal brain metastasis followed by surgical resection of the right superior lung tumor, chemotherapy with cisplatine and pemetrexed, chemotherapy to be followed by chest radiotherapy (60 Gy/2 Gy). One year later, 4 small brain metastases were treated by brain stereotaxis radiotherapy ( $24 \mathrm{~Gy}$ in a single session) and the next year new brain metastases led to panencephalic radiotherapy. Medical treatment was then switched to nivolumab, to be stopped after 2 years for severe cognitive dysfunction. The patient died 40 months after the first symptoms.

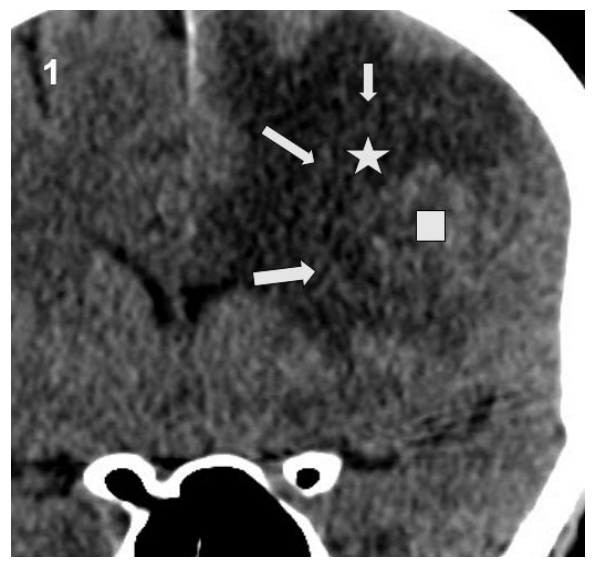

Figure 1. Unenhanced Coronal CT reformation. Frontal mass surrounded by edema. Thin peripheric wall (arrowheads). Two different densities are observed (hypodense: star and isodense: square). 


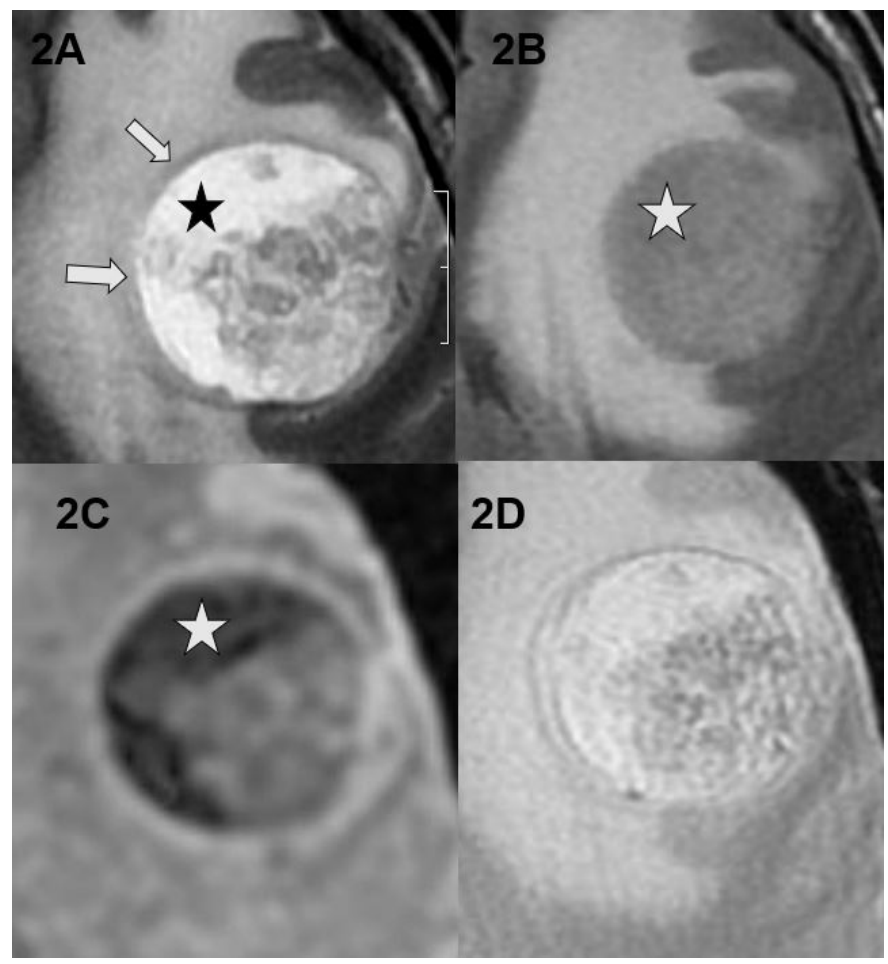

Figure 2. Axial MRI slices of the left frontal lobe. (A) T2-weighted images. (B) Fluid-Attenuation Inversion Recovery (FLAIR). (C) Diffusion-weighted image. (D) Gradient-echo image. The fluid area (star) is very hyperintense on T2-weighted imaging, isointense on FLAIR and shows presents no diffusion restriction. The gradient-echo images show neither calcification nor hemorrhage. The thin wall is isointense on T2-weighted imaging (arrows).

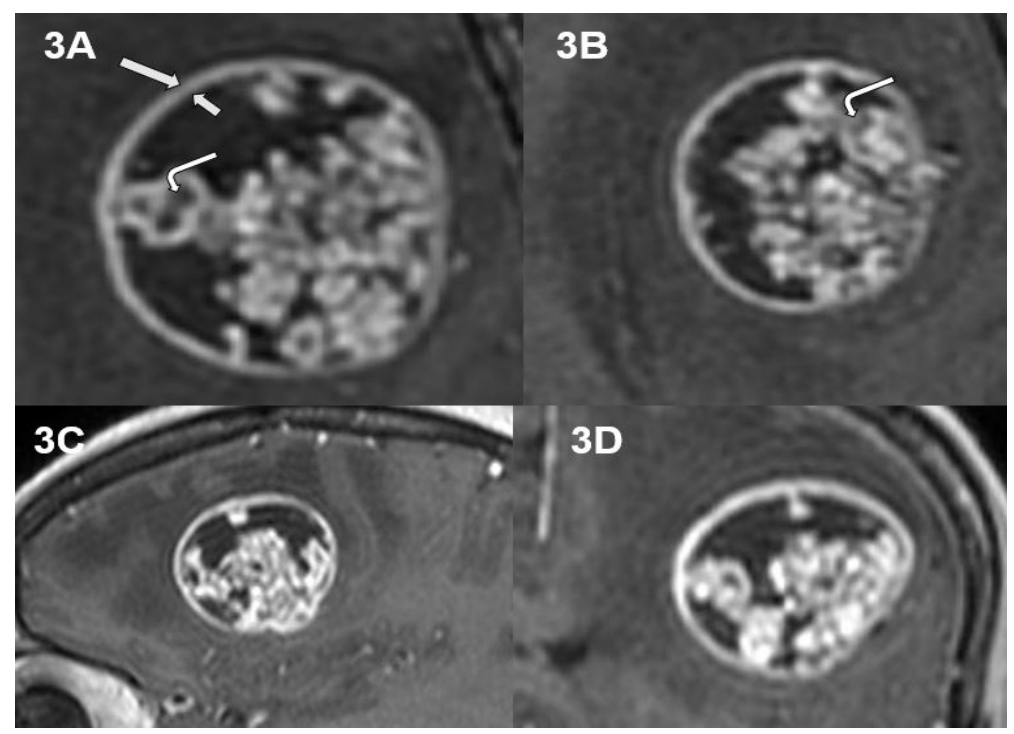

Figure 3. Axial post-contrast T1-weighted MRI (A and B). The $39 \mathrm{~mm}$-mass with regular $1.4 \mathrm{~mm}$-thick wall (straight arrows) and multiple nodular structures with hypointense centers (thin curved arrows) are seen. Sagittal (C) and coronal post-contrast T1-weighted imaging views (D). The granular structures have the same wall thickness and enhancing pattern than the outer capsule. 


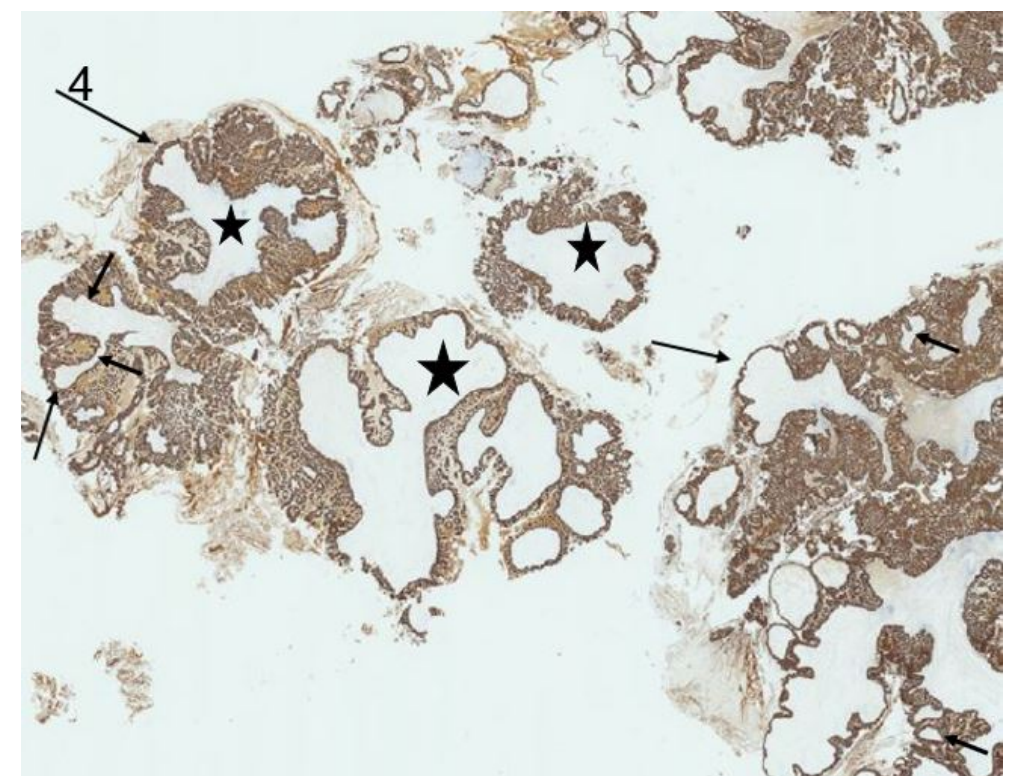

Figure 4. Histology: scan of a whole mounted slice of the resected metastasis, with mucus around small papillary tumoral nodules. These cystic nodules (star) are peripherally occupied by papillary tumoral structures with a hierarchical branching pattern: irregular papillae branch from large to progressively smaller papillae (arrows) and terminate in detached tufts of epithelial cells. The mitotic rate is low. Final diagnosis is metastasis of a lung large-cell adenocarcinoma of papillary type.

\subsection{Case 2}

The second patient is 47 year old non smoking female complaining of left upper arm transient episode of paresthesia associated with some trouble for reading and finding the correct word. Brain CT scan showed two masses mainly cystic, with some peripheral calcifications; one left temporal, the other left parietal. Parts of the cysts were filled with small irregular enhancing nodules. On MRI, there was no restriction of diffusion, the rCBV (relative Cerebral Blood Volume) was increased in the lesions, and contrast enhancement was seen after injection of gadolinium both in the capsule and in the small nodular areas of the tumor. The contrast enhancing parts of the lesions were located on the periphery of the cyst and presented a lobulated pattern, some of the small nodules enhancing only at the periphery (Figure 5(C), Figure 6(A)). Chest Xray and CT exams later found a large right upper lobe lung cancer with mediastinal nodes. Resection of the temporal lesion revealed a metastasis of a mucosecreting adenocarcinoma with papillae containing connective architecture and micropapillae without it.

Surgical resection of the left temporal and the right parietal metastases was performed after radiotherapy. Initial staging showed kidney and bilateral lung metastases. EGFR mutation was present so that Osimertinib treatment was initiated leading to a partial response on the main lung tumor, regression of the mediastinal lymph nodes and stabilization of the other lesions. The patient was stable at 12 months. 


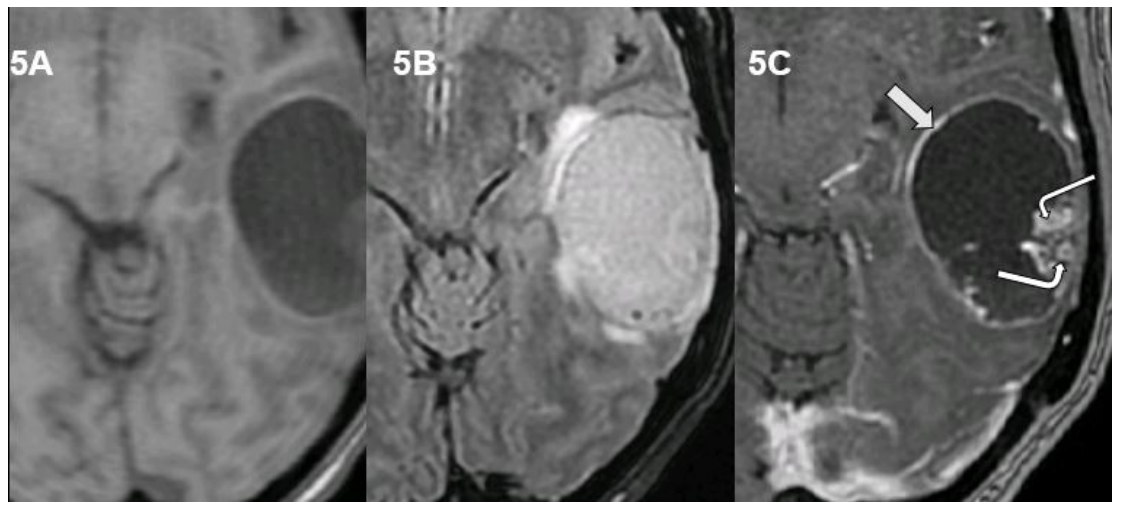

Figure 5. Patient 2. Axial MRI images of the left temporal lobe: (A) T1 non contrast, (B) T2, (C) post-contrast T1-weighted images. Enhancement of the periphery of the cystic lesion (thick arrow on (C)), and of many small nodular structures, some of those presenting with a central non enhancing area (curved arrows).

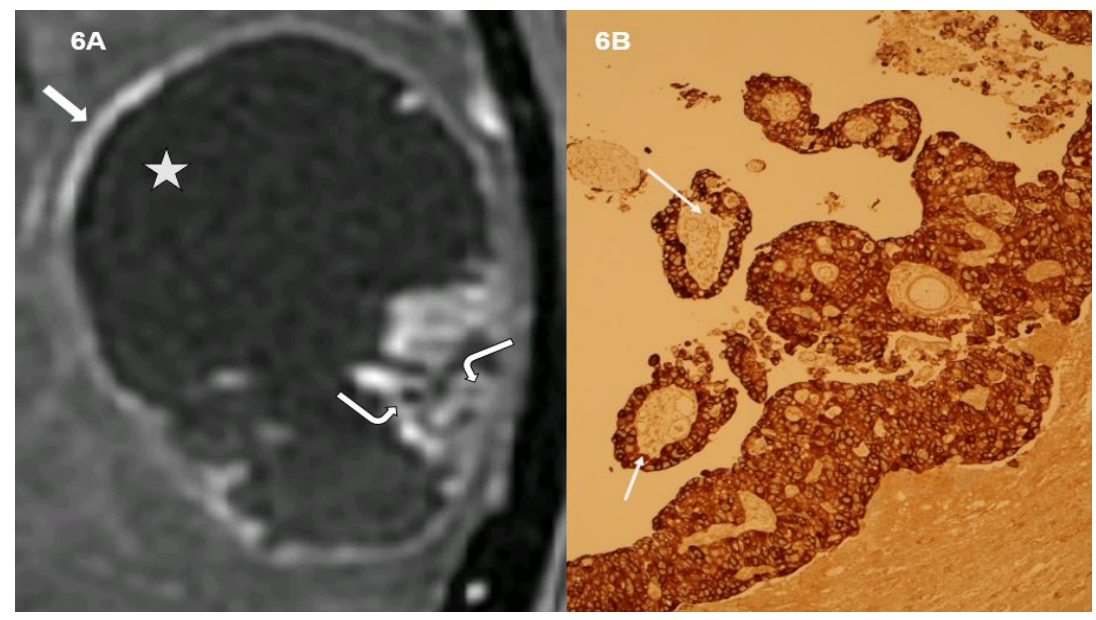

Figure 6. Patient 2. (A) Axial T1 weighted images post-contrast (central cystic area: star; enhancing peripheral wall: straight arrow; nodules with hypointense center: thin curved arrows). (B) Histology: similar papillary branched architecture in a secreting metastasis of an adenocarcinoma, probably of lung origin (irregular small papillae: thin arrows).

\section{Discussion}

Both patients had brain metastases of a lung adenocarcinoma, the brain lesions having a very similar aspect on MRI imaging: a large cystic component, a thin enhancing capsule or pseudo-capsule, and their non-cystic part was made of multiple enhancing small vegetations or papillae with some nodularity. Those nodules did not enhance homogeneously (Figure 3 and Figure 5) but sometimes had non enhancing central areas.

This peculiar MRI pattern on T1-weighted images post-injection can be closely correlated with the histologic observations of the papillary type of the adenocarcinoma, where irregular papillae branch from large to progressively smaller papillae. The non-enhancing central part of the nodules could correspond with the mucus-filled part of the papillae. 
Based on those findings, it seems that this MRI pattern has some specificity and could help the radiologist to suggest the correct diagnosis before surgery.

Brain metastases represent the most common intracranial tumors in adults. MRI imaging is very useful, spectroscopy and perfusion helping for the diagnosis of primary brain tumors while diffusion weighted images and ADC measurements may be useful to depict abscesses and lymphoma [1].

Parasitic disease must be excluded in cases of cystic lesion. "Daughter cysts" within a cystic lesion are typically described in hydatic disease, but encountered very rarely in the brain [2]. Contrast enhancement in those cases is absent, or minimal and peripheral, related to the inflammatory reaction. This is quite different from the massive and diffuse enhancement of the non cystic parts of the tumors we reported. On the other hand, cysticercus cysts are often calcified, and present as multiple enhancing cysts in a large one.

Most of the time, when infection, lymphoma and glioma are excluded, the diagnosis of metastasis is proposed without any attempt to suggest a specific origin. Some MRI patterns of brain metastases have been proved to be relatively suggestive of a specific histology: melanoma lesions were 16 times more likely than lung cancer lesions to show combined hypointensity on $\mathrm{T}^{*}$-weighted images and hyperintensity on T1-weighted images before contrast injection [3]. Metastatic brain tumors from gastric and colon cancers are frequently revealed by hypointensity on T2-weighted images, which could be due to the accumulation of collagen in the tissues [4]. Cystic metastases have been described in carcinomas of the thymus, breast, prostate, and pancreas [5]. Calcified brain metastases may be observed in patients with osteogenic sarcoma, adenocarcinoma, papillary cystadenocarcinoma, squamous cell carcinoma of the uterine cervix, ovarian carcinoma, lung and breast carcinoma [6].

Very few cases of metastases of mucopapillary lung tumor have been described in imaging: one with a miliary pattern of very small intra-axial lesions that were spontaneously hyperintense in T1-weighted imaging, mostly invisible on T2-weighted imaging and a few with hypointense spots on gradient-echo imaging [7]. Another one with the main lesion being spontaneously hyperintense on T1-weighted imaging, and partially hypointense on gradient-echo imaging [8]: those patterns associated with the multiplicity and the contrast enhancement lead to the proposed diagnosis of metastasis, melanoma being suggested in the first case [7].

The description of those metastases of a mucopapillary tumor differs greatly from our cases where the intra-axial brain tumors were seen as a partially cystic mass, with a thin regular outer wall and a large cystic component. That cystic area does not stand for necrosis but for mucus in which float solid components. The multiple small peripheral papillary structures found in the lesions strongly enhance after gadolinium injection, as does the outer wall. Some of those papillae enclose smaller cystic areas, and are sometimes bifurcated. There was no restriction of the diffusion in the cystic areas (Figure 2(C)), no hemorrhage on CT (Figure 1) and MRI. As discussed earlier, that semiology did not stand for pri- 
mary brain tumors, abscess, or parasitic disease; and the diagnosis of metastasis was suggested. There is a substantial radiologic-pathologic correlation between the T1-weighted imaging post-injection images and the pathology (Figures 3-6), suggesting that this MRI pattern has probably some specificity to the diagnosis of a papillary secreting adenocarcinoma, not specifically from the lung. The diagnosis was not proposed in the first patient, but was suggested correctly in the second case.

More research in a larger patient group is necessary to support the hypothesis that these imaging characteristics could have some specificity. Their sensitivity is clearly quite low as there were not encountered in the previously published cases.

\section{Conclusion}

We describe a striking correlation between the papillary structures found at histology and the post-contrast T1 weighted images in two cases of brain metastasis of secreting papillary lung cancer adenocarcinoma. The assumption of a specific pattern should be confirmed with larger patients series.

\section{Conflicts of Interest}

The authors declare no conflicts of interest regarding the publication of this paper.

\section{References}

[1] Pope, W.B. (2018) Brain Metastases: Neuroimaging. Handbook of Clinical Neurology, 149, 89-112. https://doi.org/10.1016/B978-0-12-811161-1.00007-4

[2] Vidhate, M.R., Singh, D., Sharma, P. and Singh, M.K. (2011) Cerebral Hydatid Cyst Showing Pathognomonic Daughter Cysts. Annals of Indian Academy of Neurology, 14, 217-218. https://doi.org/10.4103/0972-2327.85903

[3] Gaviani, P., Mullins, M.E., Braga, T.A., Hedley-Whyte, E.T., Halpern, E.F., Schaefer, P.S. and Henson, J.W. (2006) Improved Detection of Metastatic Melanoma by T2*-Weighted Imaging. American Journal of Neuroradiology, 27, 605-608.

[4] Hirano, H., Yokoyama, S., Yunoue, S., Yonezawa, H., et al. (2014) MRI T 2 Hypointensity of Metastatic Brain Tumors from Gastric and Colonic Cancers. International Journal of Clinical Oncology, 19, 643-648. https://doi.org/10.1007/s10147-013-0596-8

[5] Surov, S., Hainz, M. and Kornhuber, M. (2009) Multiple Cystic Metastases in the Brain from Adenocarcinoma of the Lung. The American Journal of Medicine, 122, e3-e4. ttps://doi.org/10.1016/j.amjmed.2009.02.030

[6] Teksam, M., Cevik, B. and Coskun, M. (2004) Calcified Brain Metastasis of Osteosarcoma: CT Findings. European Journal of Radiology Extra, 52, 47-49. https://doi.org/10.1016/j.ejrex.2004.09.007

[7] Bekiesińska-Figatowska, M., Kuczyńska-Zardzewiały, A., Klepacka, T., Brągoszewska, H., Iwanowska, B., Mądzik, J. and Lipska, I. (2013) Miliary Brain Metastases from Papillary Adenocarcinoma of the Lung-Unusual MRI Pattern with Histopathologic Correlation. Polish Journal of Radiology, 78, 57-60.

[8] Araújo, B.I.G., Teixeira, N.D., Ferreira, K., Oliveira, F.K., Ricardo, F., Salvador, G.A. and de Oliveira Ferrão, T. (2013) Brain Metastasis as Initial Presentation of Papillary Adenocarcinoma of the Lung: Case Report. Radiologia Brasileira, 46, 313-316. https://doi.org/10.1590/S0100-39842013000500008 